\title{
Adherencia a las recomendaciones de la OMS en la atención del parto y nacimiento humanizado. Medellín, Colombia
}

\author{
Adherence to the WHO recommendations in the care of \\ humanized delivery and birth. Medellin, Colombia
}

Gustavo-E Jiménez-Hernández ${ }^{1}$; Yesenia-M Peña-Jaramillo ${ }^{1}$

Forma de citar: Jiménez Hernández GE, Peña Jaramillo YM. Adherencia a las recomendaciones de la OMS en la atención del parto y nacimiento humanizado. Medellín, Colombia. Rev Univ Ind Santander Salud. 2018; 50(4): 320-327. doi: http://dx.doi.org/10.18273/revsal.v50n4-2018005 @c) (1)

\section{Resumen}

Objetivo: Describir el grado de adherencia del personal sanitario a las recomendaciones de la Organización Mundial de la Salud en la atención del parto y nacimiento humanizado en Medellín, Colombia. Métodos: Estudio descriptivo de corte transversal, se estudiaron 256 partos atendidos en tres instituciones de tercer nivel en Medellín y Rionegro Antioquia (abril - diciembre 2016), se incluyeron las gestantes que ingresaron en etapa del trabajo de parto, los datos se obtuvieron a partir de observación directa y aplicación de lista de chequeo de las recomendaciones de la OMS. Para el análisis de la información se aplicó estadística descriptiva, y para el análisis bivariado la prueba Chi cuadrado. Resultados: La proporción de cesárea fue elevada $34.8 \%$; no hubo diligenciamiento del partograma en $29,3 \%$; se viola la privacidad de las mujeres $26.2 \%$; no hubo facilidades para la deambulación $92.2 \%$; sigue presentándose el uso de fórceps, aunque en baja proporción $7.4 \%$; se realizó episiotomía en el $16.8 \%$ de los partos. Conclusiones: El grado de adherencia por parte de las instituciones y el personal de salud que participa durante el proceso de atención del trabajo de parto, parto y del recién nacido están alejadas a las recomendaciones propuestas por la OMS.

Palabras clave: Trabajo de parto; Parto humanizado; Organización Mundial de la Salud; Lista de chequeo; Asistencia; Práctica basada en la evidencia.

\begin{abstract}
Objective: To describe the degree of adherence of the health personnel to the recommendations of the World Health Organization in the care of humanized delivery and birth in Medellín, Colombia. Methods: Descriptive crosssectional study, 256 deliveries attended in three institutions of third level in Medellín and Rionegro Antioquia (April - December 2016), were studied. Pregnant women who entered the stage of labor were included, data were obtained from direct observation and application of a checklist of WHO recommendations. For the analysis of the information, descriptive statistics and for the bivariate analysis Chi square test were applied. Results: The proportion of caesarean section was high $34.8 \%$; there was no completion of the partogram in $29.3 \%$; the privacy of women is

1. Universidad Católica de Oriente-Rionegro. Antioquia, Colombia.

Correspondencia: Gustavo Jimenez. Dirección: Sector 3, Carrera 46 40B-50. Teléfono+57 45699090 Ext: 690. Correo electrónico: gjimenez@ uco.edu.co
\end{abstract}


violated $26.2 \%$; there were no facilities for ambulation $92.2 \%$; the use of forceps continues to present, although in a low proportion $7.4 \%$; Episiotomy remains an unjustified procedure with $16.8 \%$. Prophylactic antibiotics were used in $41 \%$. Conclusions: The degree of adherence on the part of the institutions and health personnel participating during the process of the care labor, delivery and the newborn are far from the recommendations by the WHO.

Keywords: Labour; Humanizing delivery; World Health Organization; Checklist; Attention; Evidence-based practice.

\section{Introducción}

La atención del parto humanizado consiste en brindar un cuidado integral, a partir de un conjunto de acciones encaminadas a una experiencia satisfactoria durante el trabajo de parto y parto. Estas actividades incluyen ofrecer un trato digno y respetuoso, que aborde lo sociocultural, emocional, y autonomía corporal, dándoles a las mujeres empoderamiento durante la atención, además, de evitar las intervenciones médicas y farmacológicas innecesarias ${ }^{1}$. Estas acciones se fundamentan en el hecho de que durante el embarazo se presentan diferentes cambios anatómicos, fisiológicos y endocrinos, y que van a tener dos propósitos fundamentales, por un lado, preparan a la mujer para el trabajo de parto y parto, protegiendo y conservando su vida, y segundo, garantizando el adecuado desarrollo y crecimiento de su bebe.

Desde la revolución industrial en el siglo XIX, se han introducido una serie de procedimientos mecánicos, uso de tecnologías y medicamentos para hacer del parto un proceso intervenido, esto con el fin proporcionar mayor seguridad y reducir las complicaciones. Los procedimientos medicalizados se incorporaron de forma gradual hasta el punto de convertirse en un ritual, por lo que muchas veces las mujeres pueden verse sometidas a maltratos y abusos afectando su salud física y mental ${ }^{2}$.

La práctica basada en la evidencia, pone al descubierto que el uso inapropiado de estos procedimientos y tecnologías durante el parto, lejos de garantizar la seguridad, ocasiona iatrogenia, aumentando la proporción de cesáreas e instrumentación del parto, obstaculizando que se constituya un buen vínculo madre e hijo ${ }^{3}$. Por tanto, se deben tener en cuenta las recomendaciones de la $\mathrm{OMS}^{4}$, que promueven prácticas que con base científica han demostrado evidentemente ser útiles y seguras, lo que incluye por ejemplo no exceder el número de cesáreas, respeto a la intimidad en el lugar del parto, apoyo afectivo por parte del personal de salud, respeto a la elección de los acompañantes durante el parto, libertad de posición y movilidad durante el trabajo de parto, evitar el uso de fármacos de forma innecesaria entre otros.
En la actualidad un gran número de mujeres a nivel mundial deben soportar un trato descortés, intolerante, humillante hasta negligente durante la atención, situación que las desmotiva significativamente, y aun no se ha concientizado en su totalidad al personal de salud sobre los cuidados y el trato adecuado que debe tenerse presente tanto en los controles prenatales, como en el momento del parto y el posparto, que permita comprender la vulnerabilidad física y mental durante esa etapa de sus vidas ${ }^{5-7}$. En la investigación que realizó la OMS sobre "Prevención y erradicación de la falta de respeto y el maltrato durante la atención del parto en centros de salud." Se evidenció que el personal de salud se caracterizó por dar un mal trato tanto físico como psicológico, constantes humillaciones, descuido en la administración de analgésicos, falta de información al momento de diligenciar el consentimiento informado, desinterés por el estado de salud de la paciente y rechazo en las admisiones a centros de salud debido a su situación económica. Infortunadamente este es uno de los grandes obstáculos que se presentan al momento de ofrecer una atención con calidad y calidez para poder brindar un cuidado humanizado.

Es por esto que la OMS insiste en que "El maltrato, la negligencia o la falta de respeto en el parto pueden constituirse en una violación de los derechos humanos fundamentales de las mujeres, descritos en las normas y los principios internacionales de derechos humanos ${ }^{8}$ "

En Colombia estas recomendaciones han tenido una incorporación lenta, se ha recomendado la implementación de una atención del cuidado exhaustivo en la práctica profesional para mantener el estado de salud de madre e hijo, aún no se ha establecido una ley para el parto humanizado como complemento de políticas y protocolos en pro del bienestar de la madre durante el proceso del parto. Sin embargo, para año 2017 el Ministerio de Salud y Protección Social emitió la circular 016 del 1 de marzo, con el fin de fortalecer acciones que garanticen una adecuada práctica para la atención segura, digna y adecuada de las maternas, que, por su condición de salud, requieran un especial trato, conforme con las rutas de atención integral, protocolos y guías de práctica clínica9. En Argentina por ejemplo, contemplan entre sus estados de gobierno la ley 25929 
para garantizar estos derechos, basados en las 16 prácticas que impone la OMS y contemplar que "el nacimiento es un proceso natural y no una enfermedad ${ }^{10}$.

En el Territorio Nacional Algunos indicadores muestran que la proporción de cesáreas está en un $45,7 \%,{ }^{11}$ considerados estos datos como epidemias de cesáreas que siguen siendo superiores a los

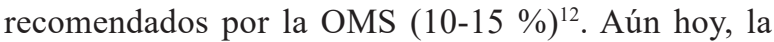
mayoría de la población y muchos profesionales de la salud piensan que no es posible dar a luz de forma segura sin el apoyo de procedimientos e instrumentos técnicos. Cuando en el proceso de atención requiera el uso de estas prácticas instrumentadas, implica que la materna debe ser informada sobre el procedimiento que se va a realizar, cuales son las razones para hacerlo, los posibles riesgos y sus beneficios ${ }^{13}$. Es importante también que al enfrentarse a tantas desigualdades e inequidades en nuestro país, las maternas en estado de vulnerabilidad por su condición social sean objeto de un mayor cuidado ${ }^{14}$.

Según Trevisan, et al. ${ }^{15}$ cuando se habla de humanización lleva inmerso un atributo de que los seres humanos son únicos e irrepetibles, por lo tanto se deben tener en cuenta las particularidades para la atención. Desde esta perspectiva, todo personal de la salud debe abordar a las personas, con un concepto holístico, como seres integrales, y una visión humanista ${ }^{15,16}$. La humanización también comprende los aspectos históricos, culturales y espirituales de los seres humanos, por lo que el personal de la salud debe determinar conexiones terapéuticas adecuadas ${ }^{17}$. El Objetivo del presente trabajo es describir el grado de adherencia del personal sanitario a las recomendaciones de la Organización Mundial de la Salud en la atención del parto y nacimiento humanizado.

\section{Metodología}

Se trata de un estudio descriptivo de corte transversal. Se registraron 256 partos/cesáreas. La información fue recolectada en tres instituciones de tercer nivel, que tienen una media de 2880 nacimientos anuales, dos de ellas son referentes en el municipio de Rionegro, y la otra institución está ubicada en la ciudad de Medellín. Los datos se obtuvieron mediante observación y el diligenciamiento de la lista de chequeo con las recomendaciones de la OMS para la atención del parto humanizado, durante la atención ${ }^{18}$, y registros clínicos. El personal sanitario que participó en la atención fue observado. Todo esto previa explicación y firma del consentimiento informado con los respectivos permisos institucionales.
La lista de chequeo de las recomendaciones incluía evaluar si se diligencia el partograma en la fase activa con una línea de acción de cuatro horas para monitoreo del avance del trabajo de parto. No se recomienda el uso temprano de la amniotomía con conducción temprana con oxitocina para la prevención del retraso del trabajo de parto, no debe inducirse el parto por conveniencia, se recomienda alentar la movilidad y adoptar una posición erguida, elegir la posición que deseen, no en posición de litotomía durante el trabajo de parto, así como el acompañamiento continuo de un familiar durante el trabajo de parto para favorecer los resultados, no se recomienda el uso de enemas, ni el rasurado de la región púbica, no se justifica la episiotomía (incisión realizada para ampliar la abertura vaginal) como procedimiento rutinario, ni el uso de fórceps, debe respetarse la privacidad de la paciente, y debe evitarse la administración rutinaria de analgésicos, anestésicos o antibióticos que no se requieran específicamente para corregir o evitar complicaciones en el parto.

La recolección de información se realizó por parte de los investigadores con el apoyo de cuatro estudiantes de séptimo semestre de enfermería, entre los meses de abril y diciembre del año 2016. El muestreo fue por conveniencia y estuvo constituido por las gestantes que fueron atendidas e ingresadas para la atención del trabajo de parto y parto entre las 7:00 am y 6:00 pm. El ingreso lo realizaba el especialista, desde ese momento se efectuó seguimiento intrahospitalario de la gestante, esto incluía recolectar las variables sociodemográficas como edad, procedencia, nivel socioeconómico, aplicación de lista de chequeo de las recomendaciones de la OMS para parto humanizado, cuyas opciones de respuesta fueron Sí/NO, y la observación directa. No hubo criterios de exclusión.

\section{Análisis de datos}

A medida que se recolectaba la información se codificaba y almacenaba en un libro de EXCEL (Microsoft office Excel 2016) por dos investigadores con el fin de evitar errores de digitación. Primero se realizó análisis con estadística descriptiva. Para las variables continuas se calcularon la media, desviación estándar, mediana o rango intercuartilico dependiendo la distribución (Shapiro Wilk). Para variables cualitativas, como las actividades que aparecen en la lista de chequeo se calcularon las respectivas proporciones, se aplicó prueba de chi cuadrado $\chi^{2}$ de Pearson para el análisis bivariado, comparando las diferencias entre lo observado y lo esperado, con los p valor. Para el análisis estadístico se utilizó el software STATA 12.0 para Windows, (Stata Corp., TX., USA) (abril 2015). 


\section{Resultados}

La mediana de edad de los partos/cesáreas estudiadas fue de 25 años (RIQ 19-32) años. La estadística descriptiva para variables materno neonatales se muestra en la Tabla 1.

Tabla 1. Estadística descriptiva para variables materno neonatales.

\begin{tabular}{lccc}
\hline \multicolumn{1}{c}{ Variable } & Media & DE & EE \\
\hline Edad & 26,07 & \pm 8053 & 0,503 \\
$<=18$ & $23,8 \%(\mathrm{n}=61)$ & & \\
$19-27$ & $36,7 \%(\mathrm{n}=94)$ & & \\
$28-36$ & $21,5 \%(\mathrm{n}=55)$ & & \\
$37-45$ & $18,0 \%(\mathrm{n}=46)$ & & \\
Edad gestacional & 38,04 & $\pm 1,74$ & 0,11 \\
$<=30$ & $1,2 \%(\mathrm{n}=3)$ & & \\
$31-34$ & $1,2 \%(\mathrm{n}=3)$ & & \\
$35-38$ & $44,5 \%(\mathrm{n}=114)$ & & \\
$39-42$ & $53,1 \%(\mathrm{n}=136)$ & & \\
Peso del Recien nacido & 2957,37 & $\pm 494,8$ & 30,92 \\
$<=750$ & $0,4 \%(\mathrm{n}=1)$ & & \\
$751-1643$ & $0,7 \% \mathrm{n}=(2)$ & & \\
$1644-2535$ & $15,0 \% \mathrm{n}=(42)$ & & \\
$2536-3428$ & $63,6 \% \mathrm{n}=(178)$ & & \\
$3429>$ & $11,8 \% \mathrm{n}=(33)$ & & \\
\hline
\end{tabular}

DE: Desviación estandar. EE: Error estandar.

Fuente: Autores

La procedencia de las mujeres fue en un $69,1 \%$ del área urbana, el 45,3 \% convivía en unión libre y según la escolaridad, tenían primaria completa en un 35,5\% Tabla 2.

Tabla 2. Variables sociodemográficas.

\begin{tabular}{lc}
\hline \multicolumn{1}{c}{ Variable } & $\mathbf{n}=\mathbf{2 5 6}$ \\
\hline Procedencia & $69.1 \%(\mathrm{n}=177)$ \\
Urbana & $30.9 \%(\mathrm{n}=79)$ \\
Rural & \\
Estado Civil & $29.7 \%(\mathrm{n}=76)$ \\
Casada & $45.3 \%(\mathrm{n}=116)$ \\
U. Libre & $25 \%(\mathrm{n}=64)$ \\
Soltera & \\
Escolaridad & $2,7 \%(\mathrm{n}=7)$ \\
Ninguna & $5,5 \%(\mathrm{n}=14)$ \\
Primaria Incompleta & $35,5 \%(\mathrm{n}=91)$ \\
Primaria completa & $30,5 \%(\mathrm{n}=78)$ \\
Secundaria completa & $9,4 \%(\mathrm{n}=24)$ \\
Secundaria incompleta & $8,2 \%(\mathrm{n}=21)$ \\
Tecnico & $8,2 \%(\mathrm{n}=21)$ \\
Pregardo &
\end{tabular}

Según las recomendaciones de la OMS, para la atención del parto humanizado, aún no se les permite acompañante durante la atención $85,9 \%$, la proporción de cesáreas fue de $34,8 \%$, se realizó episiotomía en un $16,8 \%$. Los demás ítems que constituyen las recomendaciones se muestran en la Tabla 3.

Tabla 3. Recomendaciones de la OMS para atención del parto humanizado.

\begin{tabular}{|c|c|c|}
\hline Recomendación de la OMS & SI & NO \\
\hline $\begin{array}{l}\text { Se recomienda acompañamiento } \\
\text { continuo durante trabajo de parto para } \\
\text { mejorar los resultados }\end{array}$ & $\begin{array}{l}14,1 \% \\
(n=36)\end{array}$ & $\begin{array}{c}85,9 \% \\
(n=220)\end{array}$ \\
\hline
\end{tabular}

Se recomienda el uso de oxitocina sola para el tratamiento de la prolongación $68,4 \% \quad 31.6 \%$ del trabajo de parto

$(n=175) \quad(n=81)$

Se recomienda el partograma de la fase activa con una linea de acción de $70.7 \% \quad 29.3 \%$ 4 horas para monitoreo del avance del $(\mathrm{n}=181) \quad(\mathrm{n}=75)$ trabajo de parto

No se recomienda el rasurado del area $\quad 6.3 \% \quad 93.8 \%$ pubica para el trabajo de parto $\quad(n=16) \quad(n=240)$

No se recomienda realizar enemas para reducir el uso de la conducción del trabajo de parto

$2.7 \% \quad 97.3 \%$

$(\mathrm{n}=7) \quad(\mathrm{n}=249)$

Se recomienda proporcionar $73.8 \% \quad 26.2 \%$

$\begin{array}{lll}\text { privacidad a la paciente durante el } & 73.8 \% & 26.2 \% \\ (n=189) & (n=67)\end{array}$ trabajo de parto

Se recomienda alentar la movilidad y adoptar una posición erguida durante el trabajo de parto

$(\mathrm{n}=20) \quad(\mathrm{n}=236)$

No se recomienda el uso de amniotomia sola para el tratamiento de la prolongación del trabajo de parto

$46.5 \% \quad 53.5 \%$ $(n=119) \quad(n=137)$

Parto por cesárea

$34.8 \% \quad 65.2 \%$ $(\mathrm{n}=89) \quad(\mathrm{n}=167)$

No se recomienda el uso de fórceps

$7.4 \% \quad 92.6 \%$

$(\mathrm{n}=19) \quad(\mathrm{n}=237)$

No se recomienda el uso de episiotomía $\quad 16.8 \% \quad 83.2 \%$ de forma rutinaria

$(\mathrm{n}=43) \quad(\mathrm{n}=213)$

Para el análisis bivariado, se aplicó la prueba estadística Chi cuadrado, se cruzaron las variables cesáreas y partos con las variables sociodemográficas y las recomendaciones de la OMS en la atención del parto y nacimiento humanizado. Las variables que mostraron significancia estadística para ambos eventos (cesárea/parto) fueron el rasurado de la zona púbica, la episiotomía, y el diligenciamiento oportuno del partograma con valor $p=0,001$, los demás resultados del análisis bivariado se muestran en la Tabla 4. 
Tabla 4. Analisis bivariado.

\begin{tabular}{|c|c|c|c|c|}
\hline & \multicolumn{2}{|c|}{ Cesarea } & \multicolumn{2}{|c|}{ Parto } \\
\hline & $\chi^{2}$ & p valor & $\chi^{2}$ & p valor \\
\hline Seguridad Social & 19.367 & 0,498 & 52.033 & 0,032 \\
\hline Escolaridad & 24.087 & 0,457 & 108.759 & 0,001 \\
\hline Estado Civil & 15.234 & 0,763 & 59.092 & 0,007 \\
\hline Procedencia & 6.334 & 0,176 & 7.399 & 0,389 \\
\hline Uso de enemas & 10.032 & 0,040 & 3.116 & 0,874 \\
\hline $\begin{array}{l}\text { Deambulación durante el } \\
\text { trabajo de parto }\end{array}$ & 3.332 & 0,504 & 8.501 & 0,290 \\
\hline $\begin{array}{l}\text { Se proporcionó } \\
\text { privacidad }\end{array}$ & 3.478 & 0,481 & 29.846 & 0,001 \\
\hline Rasurado de zona púbica & 24.747 & 0,001 & 19.763 & 0,001 \\
\hline $\begin{array}{l}\text { Ruptura tempana } \\
\text { artificial de las } \\
\text { membranas }\end{array}$ & 9.906 & 0,042 & 15.713 & 0,028 \\
\hline Se realizó episiotomia & 21.214 & 0,001 & 25.298 & 0,001 \\
\hline $\begin{array}{l}\text { Uso de forceps en el } \\
\text { parto }\end{array}$ & 20.055 & 0,001 & 10.290 & 0,173 \\
\hline $\begin{array}{l}\text { Diligenciamiento de } \\
\text { partograma }\end{array}$ & 30.599 & 0,001 & 29.405 & 0,009 \\
\hline $\begin{array}{l}\text { Administración temprana } \\
\text { de oxitocina }\end{array}$ & 8.999 & 0,061 & 12.236 & 0,093 \\
\hline $\begin{array}{l}\text { Acompañante durante el } \\
\text { trabajo de parto }\end{array}$ & 4.887 & 0,299 & 14.120 & 0,049 \\
\hline $\begin{array}{l}\chi 2 \text { Prueba de Chi } \\
\text { Cuadrado }\end{array}$ & & & & \\
\hline
\end{tabular}

\section{Discusión}

Para favorecer los resultados en el trabajo de parto, es necesaria una práctica basada en la evidencia con bases científicas, que permita al personal sanitario mejorar la atención, ofreciendo un trato respetuoso y aplicar intervenciones efectivas.

La OMS ha sugerido bajo la luz de la evidencia, directrices que permiten disminuir los tiempos en el trabajo de parto, evitar prácticas médicas innecesarias, de tal manera que se pueda dirigir este proceso de atención en la conducción del trabajo de parto forma más natural.

Según la OMS la realización de cesáreas es de gran utilidad en la medida en la que se empleen para salvar vidas; está establecido que no se debe sobrepasar la tasa ideal a nivel mundial la cual va de un 10 al $15 \%{ }^{19}$. Estos hallazgos muestran un comportamiento similar al reportado por el Consenso de la Federación Colombiana de Obstetricia y Ginecología (FECOLSOG) y la Federación Colombiana de Perinatología (FECOPEN).
Cuyo resultado arrojó que la tasa de cesáreas en Colombia pasó de $24,9 \%$ en 1998 a $45,7 \%$ en $2013^{11}$, el reporte del presente estudio fue de $34,8 \%$. Sin embargo, algunos autores consideran que no debe establecerse una tasa ideal dada la baja evidencia ${ }^{20,21}$. Con relación al uso del partograma, se sugiere que puede ser más útil en contextos clínicos carentes de recursos, y su uso se maximiza cuando hay una adherencia a protocolos estandarizados de la atención del parto. En dos ensayos clínicos realizados, uno de ellos en Canadá con 1156 mujeres, mostró que no hubo una diferencia significativa entre grupos (con partograma y sin partograma) (RR 1.03, IC $95 \% 0.82$ a 1.28), y otro realizado en México con 434 mujeres, evidenció un número significativamente menor de mujeres con partograma que fueron sometidas a cesárea (RR 0.38, IC $95 \% 0.24$ a $0.61 ;)^{22,23}$. En nuestro estudio el partograma se diligenció en el 70,7 \% de los trabajos de partos en fase activa.

Un estudio donde se realizó una revisión sistemática para evaluar la amniotomía temprana y el uso de oxitocina en la prevención del retraso del trabajo de parto, mostró una reducción estadísticamente significativa e importante de la duración de la primera etapa del trabajo de parto, de 4675 mujeres en el grupo de intervención tuvo un trabajo de parto más corto (DM $-1.11 \mathrm{~h}$, IC $95 \%-1.82$ a -0.41$)^{24,25}$, en este estudio se administró oxitocina en la primera etapa al $68,4 \%$ y en el 53,5\% hubo amniotomía temprana.

En muchos contextos el reposo en cama durante el trabajo de parto es usual, sin explicarle a la mujer que posición le resulte más cómoda o beneficiosa. Hay varios ensayos clínicos realizados en Reino Unido, Francia, EE.UU, Brasil, Australia, China, en la que se estudiaron 2503 mujeres, se evaluaron en posición erguidas y ambulatorias sin analgesia epidural, arrojaron que la duración de la primera etapa del trabajo de parto fue en promedio de 1,36 horas más corta (IC $95 \%-2.22$ a -0.51 ) comparadas con quienes tenían posiciones en decúbito y supina ${ }^{26,27}$. Según el reporte del trabajo realizado por Iglesias, et al. ${ }^{28}$ a las mujeres se les permitió otra postura del parto eutócico en el 74,8 $\%$. En nuestro estudio al $92,2 \%$ no se le permitió optar una posición diferente a la supina o decúbito, faltando información y soporte de alternativas relacionadas con la posición que desee adoptar la mujer durante la primera etapa del trabajo de parto.

El acompañamiento continuo de familiares o personas cercanas durante el trabajo de parto, le puede proporcionar a la mujer un apoyo significativo para afrontar los temores y preocupaciones. De hecho, en 
una revisión sistemática de Cochrane, se comparó la compañía como soporte vs. cuidados habituales, arrojó que, de 5366 mujeres estudiadas, el tiempo de duración de trabajo de parto disminuyó para quienes recibieron acompañamiento, con un tiempo promedio de 35 minutos (DM -0.58 h, IC $95 \%-0.85$ a -0.31) y la tasa de cesáreas también se redujo en un $20 \%$ (RR 0.78 , IC $95 \% 0.67$ a 0.91$)^{29}$. Nuestro reporte mostró que al $85,9 \%$ de las mujeres no se les permitió tener un acompañante en esos momentos. En nuestro contexto, por políticas institucionales existen barreras para permitir un acompañamiento permanente a las mujeres en trabajo de parto.

El uso de enemas de forma rutinaria no ha demostrado reducción del tiempo del trabajo de parto o algún otro beneficio. Es considerado invasivo y produce incomodidad en las mujeres. Un estudio realizado en Colombia donde se comparó el uso de enemas vs. el no uso, arrojó que no existen diferencias significativas en la duración del trabajo de parto (DM $28.04 \mathrm{~min}$, IC 95 $\%$-131.01 a 187.10), tampoco hubo diferencias en las tasas de traumatismo perineal (RR 0.68, IC $95 \% 0.39$ a $1.21)$, por el contrario, la infección intraparto aumentó en mujeres a las que se les aplicó enemas (RR 4.62, IC $95 \% 1.03$ a 20.68$)^{30}$. En nuestro estudio esta práctica solo se realizó en el 2,7 \% de los partos.

La episiotomía sigue siendo una práctica que se realiza de forma considerable $e^{31,32}$. En efecto un estudio de prevalencia de episiotomía realizado en Turquía, arrojó que la tasa de episiotomía fue alta, 93.3\% en mujeres primíparas y $30.2 \%$ en mujeres multíparas ${ }^{33}$, resultados por encima de los reportados en este estudio, que fue del $16,8 \%$.

Otro tipo de procedimiento en debate actual es el parto instrumentado con fórceps. Esta clase de asistencia está asociada con mayor riesgo de lesión neonatal, ocasionando hemorragia intracraneal, lesión del plexo braquial, ventilación asistida y a nivel materno, hemorragia posparto ${ }^{34,35}$. Un estudio realizado en los EE. UU, arrojó que el uso de fórceps fue del 2,7\% en los partos atendidos ${ }^{36}$, datos más bajos comparados con el presente estudio, en los que se requirió este tipo de medio en un $7,4 \%$.

\section{Conclusión}

Para plasmar una atención respetuosa y humanizada del trabajo de parto y nacimiento, es importante concienciar y transformar el proceso de atención de los servicios por parte del personal sanitario, mediante la aplicación de protocolos estandarizados resultado de la investigación profunda y en la práctica basada en la evidencia, permitiendo reducir la inconstancia de la atención.

Además, es fundamental conocer los sentimientos, las percepciones tanto de la mujer, como de su acompañante, su familia, respetando temores, culturas y creencias.

Las recomendaciones de la OMS para la atención del parto y nacimiento humanizado, tienen como propósito optimizar los resultados de las mujeres en trabajo de parto, orientada a una práctica basada en la evidencia, permitiendo así, que el personal de salud tenga herramientas para ofrecer una atención efectiva.

La adherencia por parte de las instituciones y del personal sanitario aún están alejadas de tales directrices, por parte de las instituciones, falta eliminar las barreras que impidan el acompañamiento continuo, establecer protocolos y guías de práctica clínica para el uso de adecuado de oxitocina y el diligenciamiento de partograma, permitir movilidad y posición erguida durante el trabajo de parto, no seguir implementando la amniotomía para el tratamiento de la prolongación del trabajo de parto, y, finalmente considerar el uso pertinente de la episiotomía y el fórceps. Posiblemente el grado de alejamiento del completo cumplimiento de las recomendaciones está dada por falta de implementación de estrategias y políticas institucionales que apunten a garantizar las mismas.

Las intervenciones clínicas innecesarias, traen como consecuencia el quebranto de la autonomía y dignidad de las mujeres durante el cuidado, ocasionando un impacto negativo en la experiencia del parto.

\section{Conflicto de intereses}

Ninguno declarado por los autores.

\section{Referencias}

1. Serruya SJ, Cecatti JG, Lago TDG do. O Programa de Humanização no Pré-natal e Nascimento do Ministério da Saúde no Brasil: resultados iniciais. Cad Saude Publica. 2004; 20(5): 1281-1289. doi: $10.1590 / \mathrm{S} 0102-311 \mathrm{X} 2004000500022$.

2. Uribe P, Ruiz C, Morales E. La muerte materna. acciones $\mathrm{y}$ estrategias hacia una maternidad segura. Evidencias experiencias en salud sexual y reproductiva. Mujeres y hombres en el siglo XXI. 
Vol 2. 1ed. México; 2009.

3. Nababan HY, Islam R, Mostari S, Tariqujjaman M, Sarker M, Islam MT,et al. Improving quality of care for maternal and newborn health: a pre-post evaluation of the Safe Childbirth Checklist at a hospital in Bangladesh. BMC Pregnancy Childbirth. 2017; 17(1): 402. doi: 10.1186/s12884-017-1588-x.

4. Dawe RE, Bishop J, Pendergast A, Avery S, Monaghan K, Duggan N, et al. Cesarean delivery rates among family physicians versus obstetricians: a population-based cohort study using instrumental variable methods. CMAJ Open. 2017; 5(4): E823-E829. doi: 10.9778/cmajo.20170081.

5. Silal SP, Penn-kekana L, Harris B, Birch S, Mcintyre D. Exploring inequalities in access to and use of maternal health services in South Africa. 2012; 12 : 120. doi: 10.1186/1472-6963-12-120.

6. d'Oliveira AFPL, Diniz SG, Schraiber LB. Violence against women in health-care institutions: an emerging problem. Lancet. 2002; 359(9318): 16811685. doi: 10.1016/S0140-6736(02)08592-6.

7. Small R, Roth C, Raval M, Shafiei T, Korfker D, Heaman $\mathrm{M}$, et al. Immigrant and non-immigrant women's experiences of maternity care: a systematic and comparative review of studies in five countries. BMC Pregnancy Childbirth. 2014; 14(1): 1-17. doi: 10.1186/1471-2393-14-152.

8. Organización Mundial de la Salud. Prevención y erradicación de la falta de respeto y el maltrato durante la atención del parto en centros de salud. Declaración de La OMS; 2014.

9. Ministerio de Salud y Proteccion Social. Circular 016 del 1 de marzo de 2017. Fortalecimiento de acciones que garanticen la atención segura, digna y adecuada de las maternas en el territorio nacional. 2017; 4:1-8.

10. Congreso S de sesiones del. Reglamentación de La Ley de Parto Humanizado № 25.929. Buenos Aires; 2015: 5-6.

11. Rubio-Romero JA, Fonseca-Pérez JE, Molina S, Buitrago-Leal M, Zuleta JJ, Ángel-Müller E. Racionalización del uso de la cesárea en Colombia. Consenso de la Federación Colombiana de Obstetricia y Ginecología (FECOLSOG) y la Federa. Rev Colomb Obs Ginecol. 2014; 65(2):139151. doi: 10.18597/rcog.62.

12. Bouvier-Colle M-H, Mohangoo A, Gissler M, Novak-Antolic Z, Vutuc C, Szamotulska K, et al. What about the mothers? An analysis of maternal mortality and morbidity in perinatal health surveillance systems in Europe. BJOG An Int J Obstet Gynaecol. 2012; 119(7): 880-890. doi: 10.1111/j.1471-0528.2012.03330.x.
13. Ministerio de Salud y Proteccion SocialColciencias. Guías de práctica clínica para la prevención, detección temprana y tratamiento de las complicaciones del embarazo, parto o puerperio; 2013.

14. Cáceres-Manrique FDM, Nieves-Cuervo GM. Atención humanizada del parto. Diferencial según condición clínica y social de la materna. Rev Colomb Obstet Ginecol. 2017; 68(2): 128. doi: 10.18597/rcog.3022.

15. Trevizan MA, Mendes I, Melo MR. Al encuentro de la competencia del cuidado según Boff, una nueva perspectiva de conducta ética de la enfermera gerente. Rev Latino-Am Enferm. 2003; 11(5): 652-657. doi: http://dx.doi.org/10.1590/S010411692003000500013.

16. Ibarra Coral R. Humanizacion del cuidado de enfermería durante el parto. Rev UDCA Actual Divulg Cient. 2008; 11(1) :47-56.

17. Guerrero-Ramírez R, Elisa M, Riva M-L, De M, Cruz-Ruiz L. Cuidado humanizado de enfermería según la teoría de Jean Watson, servicio de medicina del Hospital Daniel Alcides Carrión. Lima- Callao, 2015. Rev Enferm Herediana. 2016; 9(2): 127-136. doi: https://doi.org/10.20453/renh.v9i2.3017.

18. World Health Organization (WHO). Recomendaciones de La OMS para la conducción del trabajo de parto; 2016.

19. World Health Organization Human reproduction programme. WHO Statement on caesarean section rates. Reprod Health Matters. 2015; 23(45): 149150. doi: 10.1016/j.rhm.2015.07.007.

20. Souza JP, Betran AP, Dumont A, de Mucio B, Gibbs Pickens CM, Deneux-Tharaux C, et al. A global reference for caesarean section rates (C-Model): A multicountry cross-sectional study. BJOG. 2016; 123(3): 427-436. doi: 10.1111/1471-0528.13509.

21. Cyr R. Myth of the ideal cesarean section rate: commentary and historic perspective. Am J Obstet Ginecol. 2006; 194(932): 6. doi: 10.1016/j. ajog.2006.08.006.

22. Lavender T, Hart A, Smyth R. Effect of partogram use on outcomes for women in spontaneous labor at term. Cochrane database Syst Rev. 2013; 4(7): 1-79. doi: 10.1002/14651858.CD005461.pub4.

23. Weerasekara D. Usefulness of a partograph to improve outcomes: Scientific evidence. Sri Lanka J Obstet Gynaecol. 2014; 36(2): 29-31. doi:10.4038/ sljog.v36i2.7449.

24. Wei S, Wo BL, Xu H, Luo ZC, Roy C, Fraser WD. Early amniotomy and early oxytocin for prevention of, or therapy for, delay in first stage spontaneous labour compared with routine care. Cochrane 
Database Syst Rev. 2013; (8): CD006794. doi: 10.1002/14651858.CD006794.pub2.

25. Costley PL, East CE. Oxytocin augmentation of labour in women with epidural analgesia for reducing operative deliveries. Cochrane Database Syst Rev. 2012; (5): CD009241. doi: 10.1002/14651858. CD009241.pub3.

26. Lawrence A, Lewis L, Hofmeyr GJ, Dowswell T, Styles C. Maternal positions and mobility during first stage labour. Cochrane Database Syst Rev. 2009; (2): CD003934. doi: 10.1002/14651858. CD003934.pub2.

27. Gupta JK, Hofmeyr G, Shehmar M. Position in the second stage of labour for women without epidural anaesthesia. Cochrane Database Syst Rev. 2012; (5): CD002006. doi:10.1002/14651858. CD002006.pub3.

28. Iglesias Casás S, Conde García M, González Salgado S. Parto y nacimiento humanizado: evaluación de una vía clínica basada en la evidencia. Matronas Prof. 2009; 10(2): 5-11.

29. Hodnett E, Gates S, Hofmeyr G, Sakala C. Continuous support for women during childbirth. Cochrane Database Syst Rev. 2011; (2): CD003766. doi: 10.1002/14651858.CD003766.pub3.

30. Reveiz L, Gaitán HG, Cuervo LG. Enemas during labour. Cochrane Database Syst Rev. 2013; (5): CD000330. doi: 10.1002/14651858. CD000330.pub3.

31. Hsieh WC, Liang CC, Wu D, Chang SD, Chueh HY, Chao AS. Prevalence and contributing factors of severe perineal damage following episiotomyassisted vaginal delivery. Taiwan J Obstet Gynecol. 2014; 53(4): 481-485. doi: 10.1016/j. tjog.2013.07.002.

32. Hirayama F, Koyanagi A, Mori R, Zhang J, Souza JP, Gülmezoglu AM. Prevalence and risk factors for third- and fourth-degree perineal lacerations during vaginal delivery: amulti-country study. BJOG. 2012; 119(3): 340-347. doi: 10.1111/j.14710528.2011.03210.x.

33. Kartal B, Kızılırmak A, Calpbinici P, Demir G. Retrospective analysis of episiotomy prevalence. J Turk Ger Gynecol Assoc. 2017; 18(4): 190-194. doi: 10.4274/jtgga.2016.0238.

34. Gardella C, Taylor M, Benedetti T, Hitti J, Critchlow C. The effect of sequential use of vacuum and forceps for assisted vaginal delivery on neonatal and maternal outcomes. Am J Obstet Gynecol. 2001; 185(4): 896-902. doi: 10.1067/mob.2001.117309.

35. Bradley MS, Kaminski RJ, Streitman DC, Dunn S, Krans E. Effect of Rotation on perineal lacerations in forceps-assisted vaginal deliveres. Obstec
Gynecol. 2014; 122(1): 132-137. doi: 10.1097/ AOG.0b013e31829752fc.

36. Towner D, Castro MA, Eby-Wilkens E, Gilbert WM. Effect of mode of delivery in nulliparous women on neonatal. N Engl J Med. 2015;341(23): 1709- 1714. doi: 10.1056/NEJM199912023412301. 